\title{
El discurso sobre la práctica de un guitarrista de jazz semi-profesional: Estudio de caso de aprendizaje musical constructivo
}

\section{Discourse on Practice of a Semi-Professional Jazz Guitarist: A Case Study of Constructive Musical Learning}

Amalia Casas-Mas amalia.casas@unir.net Universidad Internacional de La Rioja

Ignacio Montero

nacho.montero@uam.es

Universidad Autónoma de Madrid

Juan Ignacio Pozo

nacho.pozo@uam.es

Universidad Autónoma de Madrid 


\title{
Resumen
}

Esta investigación presenta un estudio de caso de un guitarrista de jazz, aprendiz semiprofesional con un enfoque constructivista del aprendizaje. Es un caso extraído de un Estudio de Caso Múltiple, y ha sido seleccionado por su relevancia y aportación a las nuevas formas de aprendizaje musical en la sociedad actual. Nuestro objetivo es explorar su discurso sobre la práctica musical destacando las fortalezas y debilidades tanto de la cultura del jazz a la que pertenece como de su concepción del aprendizaje. Su discurso se analiza cualitativamente con un sistema estructurado de categorías deductivas e inductivas. Mostramos una descripción detallada de las condiciones de aprendizaje, resultados y procesos psicológicos a lo largo de tres fases de preparación de un tema (inicial, media y final). Hay acuerdo con las investigaciones previas, y una mejora en la descripción del aprendizaje en música popular, matizando las diferencias significativas en la cultura del jazz respecto a otras culturas, y en lo que una práctica constructiva se diferencia de otras prácticas dentro del jazz. Puede ayudar a la comprensión de las diversas formas de aprender tanto a músicos populares como clásicos.

PALABRAS ClAVE: aprendiz de jazz, constructivismo, discurso sobre la práctica, condiciones, procesos y resultados de aprendizaje

\begin{abstract}
This research presents a case study of a jazz guitarist, apprentice of high level of practice with a constructivist approach to learning. The case study draws evidence from a multiple case study and it has been selected by its relevance on what it can bring to new forms of musical learning in contemporary society. We aim to explore the guitarist's discourse about musical practice highlighting the strengths and weaknesses of both the jazz culture to which he belongs and his conception of learning. Using a structured system of deductive and inductive categories his discourse is analyzed qualitatively. We show a detailed description of learning conditions, results and psychological processes along three stages of preparing a musical piece (initial, middle and final). There is an agreement with theoretical background, and an improvement over previous research on popular music learning, qualifying significant differences in the culture of jazz regarding to other cultures, and other details of a constructive practice that differentiate it from other practices within jazz. The case study may help the comprehension of diverse ways of learning to both popular and classical musicians.
\end{abstract}

KEYWORDS: jazz apprentice, constructivism, discourse on practice, learning conditions, results and processes

\section{Introducción}

Este estudio de caso (Stake 1998) se deriva de una investigación de tesis doctoral con dos estudios empíricos sobre el análisis del discurso, con treinta y uno guitarristas participantes, estudiantes de las culturas de aprendizaje de la música clásica, el flamenco y el jazz. Además estos dos estudios (Casas-Mas, Pozo y Montero 2014; Casas-Mas, Pozo y Scheuer enviado) fueron continuados por un tercer trabajo empírico que es un estudio de caso múltiple (Stake 2006), donde se seleccionaron seis aprendices de los treinta y uno anteriores; los dos con las 
concepciones más polarizadas de aprendizaje (directa y constructiva) de cada cultura. En este tercer estudio, se observaron las prácticas musicales de aprendizaje junto con el discurso verbal en la práctica. Así podemos describir a este aprendiz en relación a los cuatro estudiantes de las otras dos culturas, en relación a las cosas que tiene en común con el otro estudiante de jazz de concepción más directa, y además tenemos referencia de las similitudes con los alumnos constructivos en las culturas clásica y flamenca de aprendizaje, así como de las diferencias con los que tenían posiciones de aprendizaje directo. No obstante, por motivos de extensión en este artículo tomamos como eje vertebrador solo el caso que aquí pretendemos ejemplificar.

Los estudios sobre las formas de aprendizaje y enseñanza de la música popular y el folklore se han incrementado de manera notable en las dos últimas décadas, así como los de comparación de éstas con los ámbitos de tradición de música clásica centroeuropea, menos numerosos. Desde las ciencias sociales se está dirigiendo la atención a los procesos que los seres humanos realizan con la música (Contreras y Hernández 2013) y esto nos está permitiendo dilucidar aspectos de la cognición que otros dominios de conocimiento no tienen la misma facilidad de activar. Así estamos accediendo, por ejemplo, a determinados procesos de autorregulación del aprendizaje, de estructuras y formas comunicativas en la interacción grupal, del uso del cuerpo y las representaciones compartidas. En este artículo, desde la psicología del aprendizaje nos proponemos analizar el discurso de un aprendiz de jazz sobre su propia práctica de aprendizaje musical. Suele plantearse la duda de si lo que se expresa verbalmente tiene algún correlato o no con lo que se lleva a cabo en la acción. Para analizarlo el objetivo en este estudio de caso es la observación del discurso situado, contextualizado a lo largo de la evolución de la práctica del aprendiz.

Varios autores (por ejemplo, Schippers 2010; Turkenburg 1999) han defendido que en la música popular el docente es sustituido por tipos de aprendizaje más autodidacta y formatos de aprendizaje entre iguales. Turkenburg (1999) sitúa el caso concreto de la música de jazz como una de las músicas populares en las que el rol del docente sirve como ejemplo al estudiante de una posible forma de hacer las cosas, pero no la única. Aunque la práctica de enseñanza actual del jazz abarca una amplia gama de aproximaciones, desde un formato muy dirigido hasta formatos exploratorios de estimulante autorregulación, sí parece tener en la base una filosofía educativa. Vamos a definir más adelante esta filosofía, fundamentada en sus orígenes y devenir histórico, tras revisar los elementos en común con otras músicas populares y tradicionales donde la improvisación juega un rol central.

En la música popular se percibe como menor la influencia de un canon "estricto" en comparación con la música clásica de tradición centroeuropea, considerada valedora de un imperialismo musical durante siglos como icono cultural con estatus simbólico (Green 2011). Aunque matizaremos luego este concepto en la cultura de aprendizaje de jazz, esto puede provocar una dificultad de definición del rol de la figura docente y esto, a su vez potenciar la actividad del rol de los aprendices, que es el que aquí nos proponemos explorar en profundidad. Green (2001) ha descrito una variedad de prácticas, actitudes y valores en el aprendizaje de la música popular, más centrados en la guitarra de rock y la música popular de la tradición anglo-americana. En sus investigaciones 
establece que las principales diferencias de este aprendizaje, en comparación con el aprendizaje más académico, se basan en el papel de la enculturación en el aprendizaje informal a través de la práctica de la escucha con atención junto con la copia (aprendizaje reproductivo). No solo radica en la disponibilidad de música grabada, sino que se combina con una observación muy cercana que siempre ha formado parte de los paradigmas del folk y la música tradicional. Esto implica que la práctica y la técnica instrumental se adquieren por mímesis en el aprendizaje.

En el ámbito de la música popular también hay un énfasis en el desarrollo del "oído", lo que implica hacer ajustes en la corteza auditiva entre la percepción y el desarrollo de las capacidades de producción, tanto instrumentales como vocales. Estas prácticas de enseñanza y aprendizaje, de escucha y copia de grabaciones por observación, pueden ser vistas como una réplica de los formatos tradicionales educativos que implican la obediencia a la autoridad del "maestro" (Campbell 1995; Lilliestam 1996), pero sin un "maestro" en vivo, lo que marca algunas diferencias que pretendemos enfatizar. Welch, Duffy, Potter y Whyton (2006/2008) sugieren además que en la música popular, en el jazz y en el folk los músicos experimentan más placer en sus actividades que sus compañeros del ámbito del clásico. En el ámbito popular, la música que se escucha en el día a día es de su propio estilo y es característico hacer música sólo por diversión.

\section{La cultura de música de jazz}

Es bien sabido que el jazz es un género musical que se desarrolló a partir de las tradiciones de África occidental, Europa y Norteamérica, podemos decir que en un proceso hibridación transcultural, usando la definición de Steingress (2004) que puntualizaremos más adelante. Fue una consecuencia de la interacción de culturas a partir del tráfico trasatlántico de esclavos entre los siglos XVI y XIX, desde las colonias africanas portuguesas, británicas, francesas, españolas y holandesas hasta el continente americano. En 1808 ya habían Ilegado cerca de medio millón de africanos subsaharianos a los Estados Unidos. Los esclavos procedían mayoritariamente del oeste de África y de la cuenca del río Congo (Cook 1999). El jazz surge muy en los principios del siglo XX, específicamente en Nueva Orleáns, Luisiana, donde Ilegaban grandes remesas de esclavos. Es por esto que el jazz nace de la combinación de tres tradiciones: la autóctona estadounidense, que propicia la fusión de los ritmos procedentes de África con las melodías y armonías de la tradición europea.

La comunidad afroamericana, en concreto la del sur de los Estados Unidos, desarrolló su expresión musical a través de la improvisación creativa sobre el material que le proporcionaban las músicas religiosas, especialmente los bailes y rituales vinculados al vudú, y también sobre material seglar propio traído de África adaptado a la tradición instrumental de las orquestas estadounidenses, sobre todo las bandas militares, y las formas y armonías de la música europea. La improvisación es ya, en estos primeros momentos, un componente esencial de estas músicas, que la contrapone a la música compuesta de los blancos. Ahora bien, este fenómeno era fundamentalmente auditivo o de tradición oral, sin ningún tipo de documentación escrita. 
Un pequeño número de músicos, principalmente de ascendencia afroamericana (por ejemplo, Buddy Bolden, Oliver Joe, Jelly Roll Morton, Bunk Johnson), sirvieron de modelo para las generaciones futuras que intentaban emular a estos músicos de Nueva Orleáns. Las actividades educativas de jazz consistieron en un principio en la escucha cuidadosa y el propósito deliberado de memorización de los aspectos claves del estilo (Murphy 1994). Las cutting sessions, y más tarde las jam sessions sirvieron como las primeras actividades "organizadas" de educación grupal en el jazz, predecesoras de las clases magistrales actuales. En ellas los músicos noveles tenían la oportunidad de aprender de otros y estaban muy enraizadas en la tradición africana del traspaso cultural vía auditiva y oral. Sirvieron como vehículo principal para la enseñanza de jazz, aún en la práctica aunque en menor grado, de hoy.

Empezaba a haber movimiento de este estilo musical en los campus universitarios en torno a los años veinte, con grupos de estudiantes que se organizaban y tocaban música para baile. Músicos de conservatorio que también tocaban jazz comenzaron a enseñar en las grandes ciudades (por ejemplo, Nueva York, Los Ángeles y Boston) y esto repercutió en la codificación del estilo. Salieron a la luz los primeros libros de métodos avalados por las principales figuras, como el de Bleihoof en 1935 (citado en Murphy 1994) y las primeras transcripciones de los solos de improvisación en revistas como Down Beat. Heinrick Schillinger enseñó improvisación y arreglos en la Schillinger House en Boston, que más tarde se convertiría en el Berklee College of Music. Lawrence Berk en 1945 fundó esta escuela que enseñaría esta música urbana popular del momento. Fue en la década de su segundo presidente, Lee Eliot Berk, cuando pasó a llamarse con el nombre actual.

Así el jazz puede ser concebido como producto de la hibridación transcultural, donde los elementos culturales cambiaron su significado o estructura semántica. El término de hibridación transcultural procede de la biología y aplicado como metáfora cognitiva en el marco de las ciencias sociales "interpreta los contactos culturales como la fuente de un sincretismo que se establece en un tercer espacio y genera un nuevo tipo de identidad y alteridad" (Steingress 2004: 187). Fue aplicado al análisis del lenguaje y finalmente la etnomusicología lo utiliza para explicar la producción de "las músicas de fusión, desterritorializadas y postétnicas" (Pelinski 2000: 156). Esta hibridación es uno de los elementos activos del cambio cultural y explica la aparición de nuevas formas culturales a partir del espíritu creativo y sintetizado de determinadas subculturas, cuyos productos "entran en la circulación internacional como lingua franca" (ibid.: 157), como ha traspasado determinado tipo de lenguaje de jazz al ámbito europeo y sus vertientes latinas. Ya han sido descritos los procesos de tensión entre los fenómenos de globalización versus los de localización (Green 2011), donde los efectos colonizadores del mundo "central" generan la construcción de las identidades musicales en relación a las músicas populares que promueven los mass media. No obstante, actualmente hay un énfasis en una nueva localización musical y nacionalismo de las regiones "peritéricas".

Actualmente el jazz que se transmite de forma académica pretende seguir los pasos de aquel que comenzó a organizarse en escuelas como el Berklee College, hasta el punto de ofrecer las mismas metodologías de ésta como un criterio social de calidad en la enseñanza. Cabe preguntarse si también sería criterio "de 
calidad" en el aprendizaje. El problema de ofrecer una metodología, a modo de receta secuenciada de pasos hasta lograr el plato deseado, es que todos los alumnos necesariamente pasan por la misma secuencia. Esto, en una cultura donde la creatividad y construcción personal parece ser un estandarte, podría producir algún conflicto en los aprendices. En ocasiones pareciera que lo que queda del origen del jazz es la idea de improvisación y las jam sessions. Lo demás en el formato educativo deriva de la institucionalización y también de una canonización del discurso (Corrado 2004/2005), que en ocasiones le hace aproximarse más a la cultura clásica y hace que se formalice y reduzcan los espacios de libertad que tenía en origen.

No obstante en el jazz, incluso en situaciones educativas de mayor institucionalización, se dan a la par que las estructuras de aprendizaje formal otras de tipo informal como las mencionadas jam sessions, fundamentales en la formación de los aprendices, analizadas en el contexto madrileño por Pedro (2014). En ellas los músicos, en un contexto musical natural (en clubes) en el que están como oyentes, se acercan al escenario (en general al mismo nivel del público) y participan tocando en la sesión en el momento en que les apetece y dejan de hacerlo de la misma manera. Este tipo de sesiones suelen tener una frecuencia semanal y son una oportunidad para tocar con músicos profesionales, disfrutar socialmente y aprender. Es un proceso posible en gran parte por el tipo de notación que utilizan, fundamentalmente standards, con melodía en notación tradicional y la armonía en cifrado, chart, de tal manera que el aprendiz cuenta con un esquema compartido de la pieza desde el principio.

En España no es hasta 1995 que se contempló por primera vez la introducción del jazz en los conservatorios, únicamente en el tramo de las Enseñanzas Superiores (Real Decreto 1995), amparado por el plan de estudios LOGSE (Ley Orgánica 1990). Desde entonces se ha implantado en los conservatorios superiores de Barcelona (ESMUC 2001), San Sebastián (Musikene, 2002), Navarra (Pablo Sarasate, 2002), La Coruña (CSMC 2006) y Valencia (CSMV 2008). En la educación superior de Madrid, la especialidad de jazz sigue impartiéndose en centros privados y municipales, con titulación propia.

\section{El aprendizaje constructivo}

Las características brevemente resumidas acerca del origen y funcionamiento de esta cultura de enseñanza y aprendizaje tiene determinados elementos que podrían relacionarse con lo que actualmente se define como concepciones constructivas, aunque también hemos definido que puede haber formatos más dirigidos, con rígidos esquemas de repetición a los que el aprendiz ha de adaptarse con la idea de "desarrollar el lenguaje". Pasamos a continuación a definir más concretamente a qué nos referimos con la concepción constructivista del aprendizaje, basándonos en Pozo, Scheuer, Mateos, y Pérez Echeverría (2006) que la definen de acuerdo a unos supuestos epistemológicos, ontológicos y conceptuales.

La propiedad sustancial de la concepción constructivista reside en sus principios epistemológicos: en este caso, se contempla la realidad como múltiple y se promueve que sea construida por el individuo, de manera que la adquisición 
de ese conocimiento implica la transformación del contenido y la del propio aprendiz. Esto conlleva diferencias en los demás principios, tanto ontológicos como conceptuales, de manera que el aprendizaje se entiende como un sistema en el cual condiciones previas, procesos de aprendizaje y resultados interaccionan en múltiples sentidos. Los componentes del aprendizaje han sido definidos por Pozo (2008): resultados (lo que aprende, lo que cambia como consecuencia del aprendizaje), procesos (actividades mentales y manifiestas que lleva a cabo el aprendiz para aprender) y condiciones (circunstancias del aprendiz y del entorno que son necesarias para que se produzca el aprendizaje).

Haciendo un resumen de lo que este tipo de concepciones constructivistas implicarían en el aprendizaje, podríamos concretarlo en los siguientes indicadores. En primer lugar, la mente del aprendiz no se concibe como un recipiente vacío, receptor pasivo del conocimiento, sino un potente instrumento activo y transformador. El aprendizaje es significativo en el momento en que enlaza con los conocimientos previos del aprendiz, lo cual dota de sentido a la tarea. Es un aprendizaje autorregulado si el aprendiz es capaz de desarrollar estrategias metacognitivas (de planificación, supervisión y evaluación) sobre su propio proceso de aprendizaje. Además los aprendices suelen estar inmersos en un formato cooperativo, donde la interacción con otros aprendices y el contexto suponen un activador de sus propios procesos cognitivos y emotivos. Finalmente, es relevante y conectado con lo todo lo anterior que este tipo de aprendices estén dirigidos hacia objetivos y metas, pero flexibles, no sujetos a un plan rígido predeterminado. El quid de la cuestión es concebir al aprendiz como un participante epistémico con un rol transformador y constructivo (Bransford, Brown y Cooking 2000; Sawyer 2006).

En relación a otras concepciones supone un cambio conceptual en el sujeto, al que, según Hofer y Pintrich (1997), contribuyen el nivel de desarrollo cognitivo tanto como el grado de conocimiento o habilidad en el contenido específico o tarea que está siendo aprendida. En el caso concreto de esta investigación, específicamente en el aprendizaje musical, el cambio conceptual también puede observarse con los diferentes usos que los aprendices hacen de la partitura. La observación de las formas y niveles de procesamiento del material representacional por parte de los aprendices constituye uno de los objetivos principales de este proyecto. En estudios previos (Bautista, Pérez Echeverría y Pozo 2010; Casas y Pozo 2008; López-Íñiguez y Pozo 2014; Marín, Pérez Echeverría y Hallam 2012) observamos diferencias en lo que los aprendices nombran en relación a la partitura y determinados niveles de procesamiento que podemos establecer.

\section{Las representaciones externas en la música}

Se definen las representaciones externas como el tipo de marcas con cualidades visuo-espaciales permanentes que sustituyen a otra realidad, en nuestro caso el sonido y el tiempo, por lo tanto lo representan. En el aprendizaje musical se han desarrollado otros tipos representaciones externas distintas a la notación y las partitura que devienen del desarrollo tecnológico y más adelante de los sistemas de grabación y de edición de sonido. Son sistemas que se despliegan en el tiempo 
en vez de en el espacio (Casas y Pozo 2008) y cuya función es fundamentalmente de regulación de la propia acción. Por un lado, sirven para registrar el conocimiento como "memoria externa", como la partitura, que es lo que algunos autores denominan función pragmática (Martí y Pozo 2000). Pero, por otro lado, los sistemas externos de representación constituyen verdaderas herramientas epistémicas, a través de las cuales no solo accedemos al conocimiento, sino con las que podríamos transformarlo y seríamos capaces de innovarlo. En los casos de aprendizaje constructivo una de las metas es la propia función epistémica de esas representaciones y no el acceso a la mera reproducción.

Las investigaciones vienen avalando la idea de distintos tipos de aproximación al aprendizaje y diferentes usos de los sistemas de notación musical en novatos y expertos. En general, los estudios sobre aprendizaje de las partituras y de la música han encontrado que los aprendices noveles suelen focalizar en micropatrones (Gruson 1988; Hallam 2007; Lane 2006; McPherson 2005), mientras que los expertos prestan atención a aspectos interpretativos y expresivos. Es como si la pericia desarrollara determinado tipo de habilidades metacognitivas de compresión de la obra musical (Gruson 1988; Hallam 2007; Hallam et al. 2012). Los niveles de procesamiento de las partituras musicales en los que basaremos el análisis en este estudio de caso fueron inicialmente abordados por Casas (2005) y Casas y Pozo (2008), y son análogos a los encontrados en otros sistemas de representación, como las gráficas y los mapas (Postigo y Pozo 2004; Pérez Echeverría, Postigo y Marin 2010). Pasamos a describirlos a continuación.

En una secuenciación jerárquica e integrada, el aprendiz hace referencia al nivel notacional al centrarse en los símbolos presentes en la partitura, es el nivel más básico de procesamiento de un material. En un nivel sintáctico el aprendiz establece relaciones entre los elementos explícitos de la partitura según una serie de reglas implícitas propias del sistema, tanto sintácticas, en un segundo nivel, como estructurales (analíticas), en un tercero. En un cuarto nivel, referencialartístico, prima la transmisión de emociones y las relaciones que el aprendiz establece entre la partitura y el estilo o época al que pertenece, el/la compositor/a que la escribió y cuestiones de interpretación ligadas a esas características estilísticas. En las investigaciones anteriormente mencionadas se analiza el uso que se hace de las notaciones musicales en el aprendizaje de la interpretación musical dentro de lo que ya podríamos Ilamar "cultura de aprendizaje musical clásica", representada aquí por los conservatorios. En ellas los niveles de procesamiento más explícitos (notacional y sintáctico) son utilizados por aprendices más noveles y los más expertos usan niveles más implícitos o referenciales.

Ahora bien, los últimos estudios apuntan a que este fenómeno no sea solo una cuestión evolutiva o de pericia, sino que en realidad hay un contexto que favorece el desarrollo de ese tipo de procesamientos más implícitos y referenciales y que además está ligado a las concepciones que mantienen los aprendices (Casas-Mas 2013) y la relación con las de sus docentes (López-Íñiguez y Pozo 2014). En estas investigaciones se muestran concepciones de tipo más realistas (directas) en las que focalizan los aprendices en mayor medida en elementos pertenecientes a los niveles más explícitos del material. Sin embargo, los aprendices con concepciones más constructivas usan niveles más implícitos o referenciales. 
Uniendo lo expuesto acerca del origen y la filosofía general de la cultura de jazz con lo tratado en este último apartado, podemos buscar algunas relaciones entre el tipo de aprendizaje de dicha cultura y esta concepción de aprendizaje, como en el trabajo de Casas-Mas, Pozo y Scheuer (enviado). En aquella investigación se definen las diferencias léxicas en relación al discurso de los aprendices de esta cultura. En ésta queremos dar el paso de ver cómo es el discurso del aprendiz cuando está situado en la práctica. Es decir, cuando nos informa lo que va a hacer, y lo que acaba de hacer. Los antecedentes en estas relaciones entre discurso y práctica (Schön 1987; Torrado y Pozo 2008), parecen indicar que efectivamente hay una distancia entre el discurso teórico y la acción, aunque se han realizado exclusivamente con docentes. Así, en este trabajo pretendemos ver el discurso situado y en un caso de aprendizaje concreto.

No hay antecedentes en el análisis del discurso sobre las prácticas musicales en un nivel tan específico como el que proponemos. De acuerdo a lo expuesto, los objetivos de este estudio de caso son los siguientes:

- Observar los procesos, los resultados y las condiciones de aprendizaje, en el discurso sobre la práctica en un guitarrista de jazz constructivo.

- Analizar con detalle los materiales y códigos representacionales utilizados por el aprendiz, bien sean notacionales, auditivos o gestuales así como el tipo de uso que hace de ellos.

\section{Metodología}

Tal y como expusimos en la introducción este es un estudio de caso extraído de un diseño ex post facto prospectivo, es decir, las culturas de aprendizaje musical de las que partimos; clásico, flamenco y jazz, representativas de ámbitos de educativos formal, informal y no-formal respectivamente (Trilla 1997; Casas-Mas 2013) en función de su estructuración e intencionalidad, lo tomamos como variable independiente no manipulable. A partir de ahí, nos propusimos observar el discurso y la práctica de aprendices pertenecientes a dichas culturas, que serían nuestras variables dependientes en la investigación. En los trabajos previos, mediante análisis de varianza y análisis lexicométrico se perfilaron las tres culturas de aprendizaje como diferentes significativamente en sus concepciones y discurso verbal. A continuación seleccionamos los dos participantes de concepciones y discurso más polarizados de cada una de las tres culturas y desde ahí implementamos un estudio de caso múltiple en el que observamos y analizamos las prácticas de aprendizaje musical a lo largo de la preparación de una pieza y el discurso situado sobre la práctica. Fueron entonces seis estudios de caso de tipo instrumental (Stake 1998) seleccionados por la variable concepción dentro de cada cultura de aprendizaje musical. Esto nos permitió observar la coherencia entre discurso, discurso situado sobre la práctica y práctica musical (Clàra y Mauri 2010). Este estudio de caso se extrae de este contexto para ser ilustrado en este artículo. 


\section{Participante}

El aprendiz ha sido seleccionado para este estudio en primer lugar por la cultura musical en la que tiene inmerso su aprendizaje, el jazz, por la razón expuesta en el marco teórico. En segundo lugar, el aprendiz fue seleccionado por el tipo de concepción de aprendizaje constructivista, a partir de dos estudios previos. La forma de selección del participante con respecto a esta variable se realizó con un procedimiento de "ida y vuelta". Partimos de su respuesta a un cuestionario (Casas-Mas, Pozo y Montero 2014) y además, analizamos sus respuestas libres mediante análisis lexicometrico (Casas-Mas, Pozo y Scheuer enviado). El análisis de las respuestas libres y cerradas de treinta y uno guitarristas semiprofesionales ${ }^{1}$ nos permitió: a) configurar el perfil léxico de la cultura de jazz, b) identificar a los participantes con concepciones más polarizadas en la elección de respuesta cerrada a los dilemas, y que además mostraron un léxico representativo de su cultura. Este aprendiz fue característico de un discurso más constructivo que el resto de aprendices de su misma cultura en los dos estudios mencionados. Así pasó a formar parte del estudio de análisis de su práctica de aprendizaje musical que exponemos a continuación.

En una breve descripción del aprendiz, mencionamos que es un varón madrileño, de veintiséis años, alumno de guitarra de jazz en la Escuela de Música Creativa de Madrid, además de estudiante de las materias de combo, armonía, etc., de final de grado profesional. Tiene la carrera universitaria de musicología y realiza el grado superior de composición. En su familia no hay antecedentes de músicos profesionales ni amateurs, solo su hermano es muy aficionado a escuchar flamenco, según su testimonio. Tiene un trío de jazz con compañeros, y toca rock y reggae en distintas agrupaciones.

\section{Material y procedimiento}

A continuación describimos el material y procedimiento llevado a cabo en este estudio. En primer lugar describimos el tema que preparó y luego las entrevistas y observación que realizamos:

Pieza musical

El tema elegido y preparado por el aprendiz fue el estándar de jazz "Lazy Bird", de John Coltrane, extraído de The Real Book (1978). John Coltrane fue músico y compositor de jazz que desarrolló su carrera especialmente entre las décadas de 1950 y 1970. "Lazy Bird" es un tema compuesto por un músico de jazz; no es un estándar de la tradición de musicales que luego los jazzistas utilizaban para improvisar, sino compuesto desde la mentalidad del intérprete en jazz. Es un tema que supone un desafío armónico para su época, con rupturas de la tradición del momento.

\section{Entrevista estructurada de preguntas abiertas}

Se recoge el discurso del aprendiz, concebido como una representación externa desplegada en el tiempo, al igual que los gestos pero con mayor definición estructural (Pérez Echeverría y Scheuer 2009), y entendido como una práctica social vinculada a sus condiciones sociales de producción, y a su marco de producción institucional, ideológica cultural e histórico-coyuntural (Pêcheux 
1978). El material de recogida de la información son entrevistas semiestructuradas de preguntas abiertas con las siguientes fases: entrevista inicial, entrevista postpráctica 1, entrevista post-práctica 2, y entrevista post-práctica 3. Las entrevistas post-práctica son similares entre sí y diferentes de la inicial, que tiene un carácter más genérico y global, porque contempla todas las fases. La práctica se dividió en tres fases: inicial, intermedia y final de la preparación completa de una pieza musical, fundamentándonos en investigaciones previas (Chaffin et al. 2003; Marín, Pérez Echeverría y Scheuer 2013; Nielsen 1999, Williamon et al. 2002). Antes de comenzar la secuencia de aprendizaje realizamos la entrevista inicial. A continuación fijamos las tres sesiones de práctica que serían observadas y grabadas a lo largo de un mes. Después de cada una de esas sesiones realizamos la entrevista post-práctica correspondiente.

\section{Resultados}

Hemos utilizado un sistema de análisis de la práctica de aprendizaje musical que, al igual que hacen otros autores en el marco de sus investigaciones, toma por componentes los elementos teóricos desde los que nuestras investigaciones vienen analizando el aprendizaje y la enseñanza de la música hasta ahora. El sistema de categorías ha sido desarrollado por el Grupo de Investigación en Adquisición de Conocimiento Musical (GIACM 2011), con un acuerdo interjueces Kappa de Fleiss $>$.80, y el pilar fundamental es que en toda actividad o práctica hay al menos tres componentes o tres preguntas a las que debemos responder (Pozo 2008).

- Qué se aprende o se pretende que se aprenda (resultados del aprendizaje).

- Mediante qué procesos y actividades aprende el alumno esos resultados, es decir, cómo se gestiona cognitiva, emocional y metacognitivamente el aprendizaje musical (procesos).

- Cómo se organizan esas actividades o prácticas (si se quiere, cómo participan profesores y alumnos en dichas prácticas y con qué materiales (condiciones).

La adaptación del sistema de análisis mencionado al estudio de este caso concreto puede consultarse en Casas-Mas (2013), donde se definen detalladamente, se justifican y se integran categorías de tipo deductivo e inductivo. Por tanto aquí ofrecemos una descripción de las categorías presentadas y explicamos los resultados que creemos de interés para la comunidad de aprendizaje y enseñanza musical. Hemos llevado a cabo un método de comparación constante (Glaser y Strauss 1967; Trinidad, Carrero y Soriano 2006), que consiste en análisis de la información empírica disponible sometiéndola a un proceso de contraste recursivo de carácter inductivo y deductivo. Para ello utilizamos el software Atlas.ti 7, que nos permite codificar las grabaciones de video de las prácticas de aprendizaje musical que así son comparadas con el resto de documentos primarios.

Presentamos los resultados del análisis del discurso sobre su práctica instrumental, divididos en tres secciones: condiciones, resultados y procesos de aprendizaje, por este orden a efectos de presentación de la lógica discursiva y complejidad. Incluimos los ejemplos textuales del aprendiz que son los que mejor 
sitúan su lenguaje original. Nosotros facilitamos una interpretación, pero a partir del texto original el/la lector/a también puede inferir sus propias conclusiones.

\section{Condiciones del aprendizaje}

Respecto a las condiciones del aprendizaje comentaremos qué tipos de materiales utiliza, de dónde extrae la información para aprender y qué hace con ellos así como la interacción que realiza con otros aprendices.

En primer lugar es relevante comentar que este aprendiz se caracteriza por un discurso muy abundante y utiliza la guitarra para ejemplificar el discurso verbal en numerosas ocasiones. Además, ocasionalmente ejemplifica con el gesto de tocar sin el instrumento y también utiliza la guitarra en situaciones que no tienen relación con el discurso verbal, a modo de desconexión (switching out) o descarga emocional (emotional discharge) (Montero y Huertas 1999).

Mediadores materiales y uso

Comenzamos describiendo las herramientas materiales que utiliza el aprendiz, es decir, las fuentes de las que extrae el conocimiento musical. En primer lugar, utiliza herramientas de audio para poder escuchar distintas versiones del tema que quiere aprender, ya sea Spotify o YouTube. Utiliza la herramienta en papel, el estándar escrito o chart, que es el esquema armónico y la melodía básica del tema. También utiliza software con secuenciación de audio y, en concreto, el Band-in-a-Box o grabaciones de Aebersold, que consisten en introducir determinado tipo de bases musicales, por ejemplo un bajo y la armonía (o ya grabadas), sobre las que el aprendiz puede tocar la melodía o improvisaciones de manera simultánea. Además, ajustándose a un ritmo establecido, porque si no lo hace no encaja con la grabación.

\section{Prácticas con esos materiales}

Es significativo que el aprendiz destaque en repetidas ocasiones la escucha de diversas versiones de manera comparativa del tema que va a aprender, con el objetivo de conformar de esta manera una versión propia, por medio del procesamiento analítico. Esas versiones las compara también con lo escrito en el chart y de ahí decide cómo va construyendo su versión. El hecho de compararlas como acción deliberada va más allá de la mera escucha y reproducción. La comprensión armónica del material en esas primeras lecturas del tema le permiten no anotar ningún tipo de función de los acordes en el papel. Esto nos indica que cuando ha comprendido e interiorizado un material, al que él hace alusión señalándose la cabeza, no anota en el papel.

Pues [lo que haría es] escucharme varias a ver cómo se tocan y sacar yo un poco el resultado para hacer la melodía, la interpretación mía de la melodía [...] lo que sí haría la semana que viene sería haber hecho un estudio de la melodía, comparativamente con distintas versiones, de cantantes, de distintos instrumentistas, un poco para hacer una interpretación propia. (e. inicial)

Además, utiliza mucho la transcripción comparativa de versiones que implican una idea de uso de la notación o escritura epistémica, es decir, no solo como mera herramienta de apoyo a la memoria sino además como proceso que le lleva 
a una comprensión y por tanto le facilita un tipo de memoria de larga duración. Esta idea la veremos reforzada con su explicación acerca de utilizar la composición (con escritura de tipo chart) como propia necesidad o gusto, más allá de la petición externa (desde el docente). Aunque pudieran parecer estrategias muy obvias, tanto el trabajo comparativo en la escucha de versiones como una escritura de tipo epistémico ninguna fueron mencionadas explícitamente por el aprendiz de jazz con concepciones más directas, en el estudio de caso múltiple.

[...H] ay veces que las escribo para contrastar cómo está escrita esta melodía e incluso hay veces que he hecho dos transcripciones melódicas de un mismo tema, dos melodías que me han gustado y que veo que son distintas sobre un mismo tema, pues las transcribo para hacer un poquito de análisis en el papel. [...A ]l escribirlo ves mucho más claro donde entran las frases, una frase que has oído mucho pues este tío la mete en una parte del compás, éste en otra. (e. post-práctica 2)

Pasamos a describir cómo usa los charts, según los niveles de procesamiento. La frecuencia con que usa el procesamiento analítico es la más alta y es un nivel en el que menciona mucho la palabra "dificultad": "[...T]rabajar los enlaces que hay de dos-cinco cromáticos, en la parte A, sí, eso yo creo que es lo más complicado de hacer [...]" (e. post-práctica 1). Lo utiliza tanto en el tratamiento del chart como para la improvisación. También destaca por un uso frecuente del procesamiento sintáctico. Es el manejo prioritario que hace del material musical. Esto nos dice que maneja mucha información condensada en esos elementos sintáctico-musicales, que le permiten descargar la memoria de trabajo: "[...H]oy es una toma de contacto, un poco leer por encima los acordes, un poquito las conexiones, las escalas, los arpegios [...] las ruedas con los voicings que haya estado mirando [...] la melodía" (e. inicial).

Hace muchas alusiones a cuestiones expresivas, que suele vincular con la textura musical, es decir, con la forma de relacionarse las diversas voces (las líneas melódicas instrumentales simultáneas) que intervienen en la pieza y que implican a otros músicos.

[...L]o más difícil de esta segunda sesión con el metrónomo es el swing, llevar el swing. Pues llevar la subdivisión correcta en el acompañamiento y la articulación de la improvisación, [...] porque hay momentos que de repente por el tipo de rueda de armonía tiendes a hacer el mismo diseño a lo mejor rítmico, entonces ahí intentar que no sea siempre lo mismo. (e. post-práctica 2)

\section{Interacción con otros aprendices}

En el estudio de caso múltiple pudimos ver que la interacción con los iguales es uno de los factores que más marcaron la diferencia entre los aprendices guitarristas de culturas populares, como jazz y flamenco y los aprendices de clásico, en los que apenas aparecían referencias al aprendizaje colectivo. Vemos que la importancia que se le da en el jazz a la textura musical hace que ese objetivo musical esté presente para la utilización de herramientas de software de secuenciación de audio, de manera que le permitan suplir las otras voces que se 
darían en una situación de aprendizaje colectivo. En su discurso explicita los componentes de responsabilidad que implica el trabajo grupal: por una parte, la composición para un grupo; por otra, la preparación de los temas compuestos por los compañeros (que valora como más difíciles que el estándar). Los problemas de gestión del grupo (p.e., que un miembro no haya trabajado individualmente lo suficiente) no los ve desde una perspectiva de carga sino que apunta más a una interdependencia positiva, es decir, no le incomoda que los temas sean difíciles, pero sabe que deben ser trabajados. Además de las materias grupales del programa de la escuela, este aprendiz se involucra voluntariamente en situaciones de aprendizaje grupal que le resultan placenteras porque tocan los temas propios. Es un elemento generador de motivación intrínseca.

\section{Resultados de aprendizaje}

En el apartado de resultados comentaremos los principales procedimientos que pone en marcha y los conceptos básicos que nombra sobre el aprendizaje y el hecho musical.

\section{Procedimientos técnicos y estratégicos}

Los procedimientos estratégicos que dice utilizar se centran fundamentalmente en la improvisación y creación con mucha frecuencia en su discurso. La improvisación y creación está muy relacionada con la comparación analítica entre distintas versiones. Mientras que en otros aprendices con concepciones menos constructivas (Casas-Mas 2013), las comparaciones implican una evaluación competitiva (es decir, que es lo que yo puedo hacer mejor o peor que otros), en este aprendiz la comparación es analítica entre versiones, previa a una toma de decisión de cómo él quiere hacerlo. La creación e improvisación también está muy relacionada con el trabajo rítmico y en especial sobre el metrónomo o a tempo. Este objetivo de mantener el pulso y el tempo tiene una estrategia ligada: tocar sin realizar paradas cuando se percibe una equivocación. La idea es aislar esas dificultades a posteriori y priorizar en la práctica el tocar de continuo aunque no salga perfecto, especialmente en la segunda sesión.

No menciona la segmentación del material, o practicar por trozos, y apenas menciona los procedimientos técnicos. Sin embargo, son frecuentemente comentados los procedimientos estratégicos en los que está implicada una toma de decisiones. Se relaciona mucho con las decisiones tomadas antes y después de improvisar. Un ejemplo de decisión previa, relacionada con el nivel de procesamiento analítico, es la de preferir tocar de continuo y aislar las dificultades más tarde. También presentamos un ejemplo de decisión a posteriori tras contemplar todas las posibilidades de acción.

Esa [versión] me sirvió poco para esto, porque es un arreglo a dúo en la que los dos van intercambiando la melodía, es todo como contrapunto, o sea no hay melodía y armonía [...] entonces a mí digamos que hacer la escala así en re siete pues digamos que tampoco me dice así tal cual el mixolidio, o sea, no consigo encontrarle feeling ahí a tocar, a lo mejor lo metería más en los mayores, donde tenga un par de compases, o en la parte B que ahí sí, un trabajo más lineal, más horizontal, pero en la parte $A$ va tan rápido que me 
decantaría más por abordar el tema motívico con arpegios. (e. post-práctica 1)

Por otra parte, es un aprendiz que incluye muchos ejemplos cantados durante todo su discurso. Sorprende totalmente ver este hecho en relación con su práctica, en la que hemos observado un canto totalmente internalizado, apenas perceptible, a partir de la sincronización de su respiración con el fraseo musical (Casas-Mas 2013).

\section{Conceptos sobre el aprendizaje}

Varias de las dificultades después de la primera sesión de aprendizaje las vinculó con el hecho de unir secciones o elementos sintácticos como las escalas. La idea de conexión de los elementos apunta a que entiende el aprendizaje como una práctica combinatoria de esos elementos, pero este tipo de práctica no la hace explícita, sino que la deducimos a partir de su explicación. Los elementos son de tipo sintáctico y son combinables en múltiples formas. Esta idea es compartida con el otro aprendiz de jazz de concepción más directa y presenta una diferencia respecto de los aprendices de las culturas de flamenco y de clásico en las que hicieron menor o ninguna referencia al proceso de aprendizaje en sí.

En los dos primeros compases hace [toca], por ejemplo, pues podríamos hacer [toca], por ejemplo, una conexión diatónica, [toca], por ejemplo una cromática ahí, notas de paso o floreos. (e. post-práctica 1)

\section{Conceptos musicales}

La relevancia del ritmo en el jazz queda patente en este aprendiz. La herramienta más mencionada es el uso del metrónomo. El objetivo de sincronización grupal parece que, en este caso, le haga priorizar mucho en sus verbalizaciones la regulación del discurso musical por medio de un elemento externo. Esto nos hace deducir que concibe el ritmo como algo que necesita ser regulado desde afuera. Este aprendiz también enfatiza el trabajo utilizando los beats en distintos puntos del compás de cuatro tiempos. Primero en los pulsos segundo y cuarto y, más adelante, con menos frecuencia, solo en el primer pulso. Explica esta estrategia especialmente para la segunda sesión y más aun en la tercera sesión.

[...P]oner el metrónomo y al dos y al cuatro [...] en este tiempo rápido pues tiendes a hacer más o menos ritmos parecidos muchas veces, entonces salir de ahí, anticipados, con retardo, a tierra; claro pero todo eso [...] pues ya es ponerlo con el tiempo, y eso es lo difícil en esta sesión, lo que intentaba en la anterior sesión era no parar, parar lo menos posible. (e. post-práctica 2)

Menciona en numerosas ocasiones el hecho de que el discurso musical vaya a tempo, hecho que contrasta aun más cuando observamos que no menciona en ningún momento estrategias de tocar lento. La música entonces vemos que se concibe desde la variable rítmica, lo que se toca ha de estar dentro de ese groove, de tal manera que tenga swing, que es lo que provoca en el oyente el movimiento, el baile. Este impulso que genera movimiento en el oyente es un resultado procedimental muy holístico que el aprendiz pretende alcanzar con una sensación de "ritmo como bailado", en sus propias palabras. 


\section{Procesos de aprendizaje}

En este apartado vamos a revisar cómo el aprendiz activa determinados procesos psicológicos que hacen posible su desarrollo en algún sentido. Este es el apartado donde más diferencias hemos encontrado entre un aprendiz de concepción constructivista y otro de concepción directa, así como en la gestión del aprendizaje muy característica en los aprendices de jazz, que pasamos a describir.

\section{Atención, memoria y aprendizaje}

De manera general, este aprendiz menciona de manera prioritaria la recuperación con transferencia sobre la recuperación literal, y el aprendizaje comprensivo sobre el repetitivo, que es característico también del aprendiz de jazz con concepciones directas y poco o nada mencionado en los aprendices de cultura clásica o flamenca. La representación mental la usa frecuentemente, hecho que tiene en común con los aprendices constructivos de clásico y flamenco y que no mencionan ni su homólogo de concepción directa como tampoco el de clásico y flamenco de concepción directa. Sin embargo, aun hablando tanto de transferencias de aprendizaje apenas hace mención al procedimiento de memorización como tal, pues éste se deriva naturalmente de la estrategia empleada. En el proceso de gestión de la atención, toma conciencia de la dificultad que supone gestionar la información en improvisación.

El proceso más mencionado es el de recuperación con transferencia, como trasladar un material que ya conoce a otros registros de la guitarra en distintas octavas, o un motivo musical cómo llevarlo a distintos tonos. También cómo aplicar algún elemento del tema a la posterior improvisación. Es relevante destacar que a él le satisface hacer ese tipo de trabajo.

También como tratamiento motívico, porque aquí la melodía siempre está con novenas, oncenas, entonces pueden ser frases sobre esas notas y transportadas en ese caso. [...H] ombre me gusta mucho esto que te he dicho antes, en cuanto a los solos, el tratamiento motívico de los dos-cinco y transportado ahí con variantes me empuja. [...E]ntonces intentar transmitir eso también al solo. (e. post-práctica 1)

Es un aprendiz consciente del uso de las representaciones mentales, fundamentalmente las de tipo visual y auditivas. Menciona y comenta cómo las utiliza y aplica, en concreto la transferencia de la visualización (o representación mental) de un dibujo en el mástil a su aplicación psicomotriz donde los dedos lo produzcan.

Utiliza mucho la alusión a la comprensión, lo que no implica que no mencione las recuperaciones literales, que suelen estar integradas en otro proceso, por ejemplo, la recuperación literal de algo que ya había creado él previamente (como son los voicings), o en un proceso de comprensión a partir de una de comparación. El aprendizaje repetitivo también es mencionado, aunque la mitad de veces que el comprensivo, más característico en jazz que en clásico o flamenco. Lo utiliza para lograr la automatización de determinadas destrezas, 
especialmente en la segunda sesión de aprendizaje. Defiende tipos de trabajo alternativos a la recuperación literal per se de las versiones de otros músicos.

Pero si, son un montón de decisiones claro, la cosa es jodida no tienes vuelta, porque ya asumes esa melodía y vale tiras para adelante, pero si la cabeza funciona más rápido puedes estar ahí a tiempo real haciendo modificaciones, que eso es lo bonito claro, y difícil de conseguir que todo eso salga natural sin estudiar ahí versiones; que siempre está bien, a lo mejor, pero no a la hora de una interpretación en directo. (e. post-práctica 2)

\section{Motivación y emociones}

El proceso de evaluación es muy habitual en él. La evaluación favorable se relaciona con emociones positivas, la motivación intrínseca, el trabajo rítmico y una observación de su propia evolución; éste es un hecho diferencial frente al aprendiz de concepción directa, cuyas evaluaciones de su propia práctica fueron más negativas y mostraban una concepción de la motivación más extrínseca, es decir, enfocada a la consecución de un "premio" (calificación, aprobación docente...) o evitación de un "castigo" (calificación, opiniones negativas...) posterior al aprendizaje.

[...Y]a después de ir pasando varias veces, vas notando que va mejor, va más suelto y eso siempre te empuja un poquito más para adelante. (e. postpráctica 1)

[...Y] eso luego lo pillo y con ganas siempre, lo cojo ahí con muchas ganas y salgo más o menos satisfecho siempre [...] Creo que bastante satisfecho [...] evidentemente es una tercera sesión, que para tocar en un concierto haría falta un poco más, pero estoy contento con la evolución y con cómo lo he estudiado. (e. post-práctica 3)

Las emociones también podemos observarlas en su discurso. Al mencionarlas prioriza la búsqueda de emociones positivas como objetivo de su trabajo, a diferencia del aprendiz directo, y expresa con ellas aquello que le gusta y que le emociona, como vimos en el ejemplo.

[...M]e moló mucho ver la melodía cómo está hecha, es una melodía así en un ámbito muy cortito y cómo pasa por todos estos acordes que son notas muy chulas en las que reposa. (e. post-práctica 1)

El ratito que estuve, estuve cómodo, estuve pasándolo bien... (e. post-práctica 2)

Las atribuciones que realiza tienen el locus de control interno y modificable, es decir, la causa de que algo no le haya salido bien la ubica en un procedimiento en concreto que él puede realizar junto con el control de procesos, como la gestión de la atención o la regulación del estudio. Por todo lo que acabamos de explicar en otros procesos, deducimos que su motivación es fundamentalmente intrínseca, centrada en su relación con la música y su propio aprendizaje. Es motivación intrínseca porque se centra en su propia evolución y proceso de aprendizaje, no está condicionada a efectos positivos o negativos posteriores al mismo. 


\section{Gestión del aprendizaje y autorregulación}

En primer lugar, es el alumno quien realiza la elección de la pieza y argumenta razones de textura instrumental y reto de aprendizaje. No menciona la intervención del profesor en la elección de los temas. Habla de autonomía en el aprendizaje "siempre me motiva estudiar por mi cuenta" (e. post-práctica 3), siendo uno de sus elementos motivadores. No alude a la gestión externa del docente en el aprendizaje.

En segundo lugar, estamos ante un aprendiz que se centra fundamentalmente en la planificación y regulación del estudio. Así, se centra en distintas prioridades por sesiones: en la primera, la cohesión del tema evitando las paradas; en la siguiente, salir de formatos repetitivos y promover la variación dentro del trabajo rítmico con metrónomo. Además establece una temporalización de trabajo por días de la semana. Durante el fin de semana intenta trabajar también, aunque vemos que prioriza los formatos de música colectiva, como es un grupo en el que tocan sus propios temas o los conciertos en fin de semana.

Los objetivos hacen referencia a lo que decide tocar según las texturas musicales-instrumentales que quiere conseguir. Vemos que aunque en primera instancia es un tema para tocar a dúo, no se limita a la preparación del tema para ese formato, sino que piensa en posibilidades de más y menos componentes.

[...P]orque el fill en este caso concreto nada más en este momento que estoy trabajando yo con la melodía, el fill sería más de otros instrumentos, yo creo, o del acompañante, también eso cómo lo arregles o con quién lo toques. (e. post-práctica 1)

A continuación, revisamos los momentos del discurso en que menciona las dificultades que se encuentra. Lo hace muy frecuentemente y vemos que evoluciona según la sesión en la que se encuentra. En la entrevista inicial, el aprendiz prevé cuáles serán sus dificultades en la primera sesión, relacionadas con la armonía del tema. Coinciden con lo que él explica en la entrevista postpráctica 1.

[...] hay un par de ruedas de acordes que voy a tener que estudiar, jaja [...] es de dificultad media-alta porque tiene ahí esos cambios, que a lo mejor con el dos-cinco no resuelven. (e. inicial)

Al final de la entrevista post-práctica 1 ya empieza a prever que las dificultades en la segunda sesión serán de improvisación en el tempo del tema, con velocidad. En la segunda sesión se corrobora esta dificultad, y en ella se dirige más a variar el material musical y a tomar decisiones para crear en tiempo real, ya que la velocidad del tema es alta. $Y$ sugiere tipos de práctica para resolverlo: "[...E]ntonces eso lo tocas con la versión, o con un secuenciador, bien" (e. postpráctica 2).

En la tercera sesión, la dificultad se centra más entre la representación que él tiene musical en su "cabeza" y la visualización del dibujo a realizar por los dedos sobre el mástil. El aprendiz tiene la intención de centrarse más en la resolución de detalles que la idea holística de continuidad que tenía en la primera sesión. Y se apoya en otras personas para resolverlo: "Hay veces que me veo en bucles 
metido también un poco que intento corregir, o hablar con alguien ahí que me ayude salir de una cosa en concreto" (e. post-práctica 3).

Este tema el aprendiz lo había clasificado como de dificultad media-alta y aún así clasifica de "más difíciles" los temas que componen los compañeros. Esto puede ser porque sean menos estándar y por tanto menos previsibles. El apoyo en el grupo es una estimulación de aprendizaje y, en este nivel avanzado, puede darse que en otras culturas los aprendices no opinen lo mismo de las obras compuestas por sus iguales respecto a las de los compositores emblemáticos.

[...L]levaba un ensayo de un cuarteto que estábamos ahí montando temas de audiciones de Pamplona de los chicos, del bajista y el batería, y claro pues todo bien a primera, pero no son temas tipo "Lazybird", son un poco complejos. (e. post-práctica 3)

Terminamos la presentación del discurso de este aprendiz con los ejemplos en los que utiliza los materiales musicales adaptándolos para sí. No hay ninguna mención explícita al proceso de que él se adapte al material externo, más allá de los ajustes al tempo regulado por el metrónomo que hemos comentado. No obstante, ese ajuste a la velocidad no le coarta el hacer sus propias versiones e improvisaciones de la música según a él le va pidiendo.

[...C]on la melodía no tuve problemas, porque la varié ya el otro día y no la tocaba ya como está aquí escrita. Al principio sí lo hice [tocar con las duraciones que están escritas] al principio un par de vueltas, pero enseguida ya me pide otra cosa. (e. post-práctica 1)

La elección de la pieza por el alumno, la autonomía del aprendizaje, la escasa o nula gestión del profesor, la planificación desarrollada del trabajo, una detección de dificultades muy alta y un ajuste del material externo a sus necesidades de aprendizaje (en vez del ajuste personal al material), son las características más remarcables de lo encontrado en el discurso de los dos aprendices de jazz y que los diferencia de los de clásico y flamenco. Quiere decir, que muestran un perfil mucho más autorregulador, en terminología de Sokol, Grouzet y Müller (2013) que sus compañeros de las otras dos culturas. En la TABLA 1 ofrecemos un resumen de los aspectos más característicos descritos acerca del discurso sobre la práctica de aprendizaje musical de este guitarrista de jazz con concepción de aprendizaje constructivista. 
TABLA 1. Resumen de los aspectos más relevantes del discurso sobre la práctica del aprendiz de Jazz constructivo (Casas-Mas 2013).

\begin{tabular}{|c|c|c|}
\hline \multirow{3}{*}{ 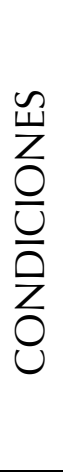 } & $\begin{array}{l}\text { Mediadores materiales y } \\
\text { uso }\end{array}$ & $\begin{array}{l}\text { - Proceso comparativo de versiones } \\
\text { - Saca de oído y compara } \\
\text { - Transcripción comparativa } \\
\text { - Usa el metrónomo principalmente } \\
\text { - Lectoescritura epistémica }\end{array}$ \\
\hline & $\begin{array}{l}\text { Prácticas con los } \\
\text { materiales }\end{array}$ & $\begin{array}{l}\text { - Procesamiento analítico y sintáctico elevados } \\
\text { - Procesamiento expresivo alto } \\
\end{array}$ \\
\hline & $\begin{array}{l}\text { Aprendizaje colectivo } \\
\text { (pares) }\end{array}$ & - Alto, interdependencia \\
\hline \multirow{3}{*}{ 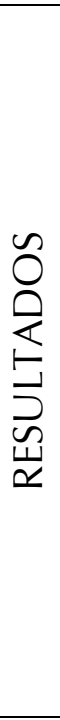 } & Procedimientos & $\begin{array}{l}\text { - Crear e improvisar } \\
\text { - No segmentación del material } \\
\text { - Muchos estratégicos } \\
\text { - Cantar mucho en discurso y nada en la } \\
\text { práctica } \\
\text { - Psicomotrices un poco } \\
\end{array}$ \\
\hline & $\begin{array}{l}\text { Conceptos sobre el } \\
\text { aprendizaje }\end{array}$ & $\begin{array}{l}\text { - Por conexiones } \\
\text { - Error como punto de partida } \\
\end{array}$ \\
\hline & Conceptos musicales & $\begin{array}{l}\text { - Ritmo con metrónomo mucho } \\
\text { - Ritmo a tempo bastante } \\
\text { - Ritmo bailado } \\
\text { - No tocar lento } \\
\text { - No menciona cualidades del sonido, pero } \\
\text { saca mucho de oído y compara versiones }\end{array}$ \\
\hline \multirow{3}{*}{ 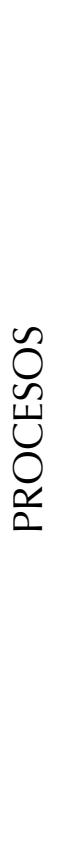 } & $\begin{array}{l}\text { Memoria y recuperación } \\
\text { de información }\end{array}$ & $\begin{array}{l}\text { - Transferencia mucha } \\
\text { - Poca recuperación literal } \\
\text { - Mucha comprensión } \\
\text { - Repetición } \\
\text { - Representación mental }\end{array}$ \\
\hline & Motivación y emociones & $\begin{array}{l}\text { - Atribuciones (más positivas) } \\
\text { - Evaluación mucha, más positiva } \\
\text { - Motivación intrínseca } \\
\text { - Emociones positivas }\end{array}$ \\
\hline & $\begin{array}{l}\text { Gestión del aprendizaje y } \\
\text { autorregulación }\end{array}$ & $\begin{array}{l}\text { - Elección pieza alumno } \\
\text { - Autonomía en aprendizaje } \\
\text { - Ninguna gestión del profesor } \\
\text { - Planificación bastante } \\
\text { - Objetivos } \\
\text { - Regulación del estudio alta } \\
\text { - Detección dificultades muy alta } \\
\text { - Adapta material a sí mismo }\end{array}$ \\
\hline
\end{tabular}




\section{Discusión}

Un estudio de caso tiene una intención ideográfica y no nomotética, es decir, no generalizable por ley a todos los miembros de una cultura. Es así desde dónde realizamos la discusión de los siguientes aspectos encontrados en el análisis del discurso sobre la práctica musical en este aprendiz de jazz constructivo. Para discutir el primer objetivo propuesto organizamos la presentación de acuerdo a los componentes tratados: las condiciones, los resultados y los procesos de aprendizaje.

En referencia a las condiciones de aprendizaje, hemos encontrado en este aprendiz el perfil descrito por Green (2001) para el aprendizaje de la música popular, basado en la enculturación (Howe y Sloboda 1991), donde prevalecen la escucha con atención y copia, que, en palabras de la autora, desarrollan el oído. En el caso de este aprendiz observamos que predomina esa aproximación auditiva al material musical, pero podemos decir que va más allá de un formato meramente reproductivo, desde el momento en que realiza escuchas y transcripciones comparadas de versiones. El tipo de aproximación a la música además concuerda con el devenir histórico de la tradición jazzística (Murphy 1994) en las que las primeras actividades educativas se basaban en la escucha cuidadosa y la memorización de los aspectos claves del estilo, con ayuda de la transcripción. Además, también este aprendiz alude al apoyo del aprendizaje colectivo, como el derivado de las jam sessions. En el estudio de caso múltiple (Casas-Mas 2013) fue Ilamativo el hecho de que los aprendices de jazz fueron los únicos que antes de comenzar a practicar hicieron escuchas de varias versiones. Tanto en la cultura de aprendizaje clásica como flamenca se limitaron a la escucha de una única versión de referencia. En el caso del aprendiz constructivo de jazz, además hizo explícita la práctica comparativa entre ellas. El aprendizaje colectivo y grupal no fue relevante para los aprendices de clásico.

El participante de jazz de este trabajo, como suele ser habitual, inicia su formación en esta cultura cuando ya tiene una base de formación clásica. Pone mucho énfasis en los procesamientos analítico y sintáctico del material musical, en línea con el trabajo de Hallam et al. (2012), que enuncia que el conocimiento armónico y formal generalmente se añade en la enseñanza a partir de los niveles intermedios. Esto nos hace pensar que la complejidad en el análisis de una obra es vista como un factor importante en la interpretación y práctica musical, como indica el estudio de Vaughan (2002). Es por esto que podemos apreciar una tradición que ha seguido más la estela del academicismo de las primeras escuelas universitarias de Boston que los formatos en que se originó, que eran más cercanos al aprendizaje informal de herencia africana.

Esto supone que el aprendiz se encuentra ya más cercano a una aproximación a la música simbólica-explícita que a una aproximación basada en la acciónimplícita, que conlleva la disociación cuerpo/mente. No obstante, dentro de la disociación que puede conllevar un formato académico, en este aprendiz hemos podido constatar la satisfacción y placer que le produce su aprendizaje, que llega a identificar con la experiencia de flujo (Csikszentmihalyi 1975, 1990), apoyando lo encontrado en música popular por Hytönen-Ng y Hurley (2013) y por el proyecto de Welch et al. (2006/2008). Es importante a la hora de comentar su aproximación, que manifiesta un movimiento (o motivación) intrínseco centrado 
en el propio proceso de aprendizaje. López-Íñiguez y Pozo (2014) enunciaron factores de influencia en la construcción de esa concepción intrínseca tales como las relaciones con otros agentes educativos y el entorno de aprendizaje.

En relación a los resultados de aprendizaje, de todo lo analizado destacamos el concepto y tratamiento del pulso, del tempo y del ritmo, parámetros muy importantes en músicas distintas a la de la tradición clásica centroeuropea. Este hecho lo hemos visto relacionado con el uso que hace este aprendiz de representaciones externas que se despliegan en el tiempo y responde al segundo objetivo de este proyecto. Es lógico pensar que en la música popular hay un énfasis en la sincronización rítmica, coincidiendo con un énfasis en el aprendizaje colectivo. Creemos que tanto los elementos sociales como los rítmicos son los que pueden posibilitar cambios en el uso de múltiples mediadores, artefactos teóricos y prácticos, en el aprendizaje musical en los ámbitos más académicos. Son referentes de aprendizaje que nos aporta la música popular. Y el uso que el aprendiz hace de tales representaciones externas coincide con lo que otros autores (Gruson 1988; Hallam 2007; Lane 2006; McPherson 2005) han definido como comportamiento experto; centrado en aspectos interpretativos en las primeras sesiones y en micro patrones casi en el final de la preparación, más abundantes en la entrevista que realizamos después de la tercera sesión de práctica (post-práctica 3). Aunque, de nuevo desde nuestro punto de vista, lo relacionaríamos con el tipo de concepción constructiva del aprendiz y no exclusivamente asociado a una cuestión de pericia (Casas-Mas 2013).

Respecto a los procesos de aprendizaje observados destacamos aquí los referentes a la autonomía del aprendiz (Sokol et al. 2013) coincidiendo con los trabajos sobre música popular (Casas-Mas, Pozo y Scheuer enviado; Shippers 2010; Turkenburg 1999). Vemos que coincide con lo que Pozo et al. (2006) definían como propiedad consustancial de la concepción constructivista al describir la realidad como múltiple y en la que el aprendiz toma una serie de decisiones para construir su propia visión del fenómeno musical. Además, es crucial el hecho de primar procesos de transferencia del conocimiento y la comprensión versus la mera repetición. Sin embargo, en la música popular seguramente podemos mostrar matices a esta afirmación, o gradientes de autonomía; como vemos en Casas-Mas, Pozo y Montero (2014), esta variable está muy diferenciada entre el jazz y la cultura de flamenco, e incluso diferenciada dentro del mismo jazz en el caso de contextos con concepciones más reproductivas o directas. Tal y como defienden algunos autores (Bathgate, SimsKnight y Schunn 2012), podríamos aplicar las conclusiones de este trabajo para promover que los estudiantes sean estimulados a adoptar estrategias metacognitivas y analíticas que les faciliten "la autonomía" durante la práctica de aprendizaje instrumental. El quid de la cuestión es cómo enfatizar esa autonomía que genera aprendizajes tan eficaces sin propiciar una desconexión símbólicoacción o si se prefiere racional-emoción ¿Son reconciliables las dos aproximaciones?

Sloboda (2010) definió la "experiencia de concierto clásico" en la que puede alcanzarse un éxtasis de comportamiento con apenas expresión emocional ni movimiento por parte de los oyentes. En el caso de la cultura de jazz está establecido el aplauso después de los solos de improvisación, que sirven por una 
parte de inclusión de la participación del oyente, y de interacción y retroalimentación de la interpretación del intérprete por otra. No obstante, si bien esta "licencia" marca una diferencia de canon con la cultura de música clásica, en el jazz suceden tanto en oyentes como en intérpretes experiencias "intelectuales" que en ocasiones se describen más centradas en la coherencia interna del discurso musical que en la forma de comunicación del mismo. Parece que los músicos de jazz adquieren y activan un conocimiento procedimental que encarna los principios que rigen la armonía, métrica, ritmo, y el contorno melódico (Berliner 1994; Johnsson-Laird 2002) y les permite improvisar en tiempo real. Esto es producto de muchas horas de trabajo, pero también de la cualidad de ese tipo de trabajo en el que el aprendiz tiene constantemente que estar mirando "hacia adentro" y ajustando el material a sus capacidades en un determinado momento y no al revés (Casas-Mas 2013). Desde un punto de vista de procesos psicológicos podríamos decir que se establece una diferencia considerable con otros formatos y culturas de aprendizaje reproductivo. Pero, este artículo nos permite también diversificar una visión unitaria de la cultura de aprendizaje de jazz (de discursos y prácticas reproductivas a más constructivas) y tomar perspectiva, atendiendo a lo que Ochoa (2010) describió como los discursos de superioridad del jazz frente a otras músicas populares contemporáneas. Vemos matices en las luces, pero también en las sombras del énfasis de cualquier concepción de aprendizaje, que siempre suponen un hincapié en determinado tipo de prácticas en detrimento de otras. Hay momentos en que las culturas toman distancia del origen en que surgieron, y este fenómeno suele provocar la alternancia con tendencias a volver al mismo. Estas tendencias vienen a denominarse, tanto en música como en psicología, como hot. La reflexión escrita nos permite preguntarnos de estas últimas tendencias en hot cognition y hot jazz el por qué de esa necesidad.

\section{Notas}

${ }^{1}$ Como ponen de manifiesto los trabajos de Green (1997) y Lorenzo Arribas (2011), en la música popular hay un sesgo de género hacia las mujeres instrumentistas. En los participantes de jazz solo conseguimos a una mujer guitarrista en este nivel semiprofesional.

\section{Agradecimientos / Acknowledgements}

Parte del material en este artículo se basa en la investigación de las tesis Culturas de aprendizaje musical: concepciones, procesos y prácticas de aprendizaje en clásico, flamenco y jazz, por Amalia Casas-Mas (2013).

Some of the material in this article is based on the research for the thesis Musical Learning Cultures: Learning Conceptions, Processes and Practices in Classical, Flamenco and Jazz by Amalia Casas-Mas (2013). 


\section{Referencias}

Bathgate, M., Sims-Knight, J. y Schunn, C. D. 2012. Thoughts on Thinking: Engaging Novice Music Students in Metacognition. Applied Cognitive Psychology 26 (3): 403-409. doi: 10.1002/acp.1842.

Bautista, A., Pérez Echeverría, M. P. y Pozo, J. I. 2010. Music Performance Teachers' Conceptions about Learning and Instruction: A Descriptive Study of Spanish Piano Teachers. Psychology of Music 38 (1): 85-106.

Berliner, P. 1994. Thinking in Jazz: The Infinite Art of Improvisation Chicago: University of Chicago Press.

Bransford, J. D., Brown, A. y Cooking, P. 2000. How People Learn: Brain, Mind, Experience, and School. Washington: National Academic Press.

Campbell, P. S. 1995. Of Garage Bands and Song Getting: The Musical Development of Young Rock Musicians. Research Studies in Music Education 4: $12-20$.

Casas, A. 2005. Formas de notación externa en la enseñanza y aprendizaje de la música. Memoria presentada para la obtención del DEA, manuscrito no publicado. Universidad Autónoma de Madrid.

Casas, A. y Pozo, J. I. 2008. ¿Cómo se utilizan las partituras en la enseñanza y aprendizaje de la música? Cultura y Educación 20 (1): 49-62. doi: 10.1174/113564008783781503.

Casas-Mas, A. 2013. Culturas de aprendizaje musical: concepciones, procesos y prácticas de aprendizaje en clásico, flamenco y jazz. Tesis Doctoral. Facultad de Psicología. Universidad Autónoma de Madrid, España. https://repositorio.uam.es/handle/10486/14310

Casas-Mas, A., Pozo, J. I. y Montero, I. 2014. The Influence of Music Learning Cultures on the Construction of Teaching - Learning Conceptions. British Journal of Music Education 31 (3): 319-342. doi:10.1017/S0265051714000096 .

Casas-Mas, A, Pozo, J.I. y Scheuer, N. enviado. Musical Learning Conceptions as Sociocultural Productions. Lexicometrical Analysis of Discourse in Classical, Flamenco and Jazz Cultures.

Chaffin, R., Imreh, G., Lemieux, A. y Chen, C. 2003. Seeing the Big Picture: Piano Practice as Expert Problem Solving. Music Perception 20: 465-490.

Clàra, M. y Mauri, T. 2010. Una discusión sobre el conocimiento práctico y sus relaciones con el conocimiento teórico y la práctica. Infancia y Aprendizaje 33 (2), 199-207.

Contreras, I. y Hernández, S. 2013. Pensar la música desde las ciencias sociales. Entrevista a Esteban Buch. TRANS-Revista Transcultural de Música/Transcultural Music Review 17: 1-35.

Cooke, M. 1999. Jazz. London: Thames and Hudson.

Corrado, O. 2004/2005. Canon, hegemonía y experiencia estética: algunas reflexiones. Revista Argentina de Musicología 5-6, 17-44.

Csikszentmihalyi, M. -

1975. Beyond Boredom and Anxiety: Experiencing Flow in Work and Play. San Francisco: Jossey-Bass

1990. Flow: The Psychology of Optimal Experience. New York: Harper and Row. 
Glaser, B.G. y Strauss, A. 1967. The Discovery of Grounded Theory: Strategies for Qualitative Research. Chicago: Aldine.

Green, L. -

1997. Music, Gender, Education. Cambridge: Cambridge University Press.

2001. How Popular Musicians Learn: A Way Ahead for Music Education. Aldershot: Ashgate Press.

Ed. 2011. Learning, Teaching, and Musical Identity. Voices across Cultures.

Bloomington: Indiana University Press.

Grupo de Investigación en Adquisición de Conocimiento Musical (GIACM). 2011.

Sistema de análisis de las prácticas de aprendizaje/enseñanza en música

instrumental. Documento no publicado. Departamento de Psicología Básica.

Universidad Autónoma de Madrid, España.

Gruson, L. M. 1988. Rehearsal Skill and Musical Competente: Does Practice Make Perfect? En J. A. Sloboda Ed., Generative Processes in Music: The Psychology of Performance, Improvisation, and Composition. New York: Oxford University Press: 91-112.

Hallam, S. 2007. Music Psychology in Education. London: Institute of Education, University of London.

Hallam, S., Rinta, T., Varvarigou, M., Creech, A., Papageorgi, I., Gomes, T. y Lanipekun, J. 2012. The Development of Practising Strategies in Young People. Psychology of Music 40 (5): 652-680. doi: 10.1177/0305735612443868.

Hofer, B. K., y Pintrich, P. R. 1997. The Development of Epistemological Theories: Beliefs about Knowledge and Knowing and Their Relation to Learning. Review of Educational Research 67 (1): 88-140.

Howe, M. J. A. y Sloboda, J. A. 1991. Young Musicians' Accounts of Significant Influences in their Early Lives. 1. The Family and the Musical Background. British Journal of Music Education 8: 39-52. doi:10.1017/S0265051700008056.

Hytönen-Ng, E. y Hurley, A. W. 2013. Experiencing "Flow" in Jazz Performance. IASPM@Journal (4) 1:124-125.

Johnson-Laird, P. N. 2002. How Jazz Musicians Improvise. Music Perception 19 (3), 415-442.

Lane, J. S. 2006. Undergraduate Instrumental Music Education Majors' Approaches to Score Study in Various Musical Contexts. Journal of Research in Music Education 54: 215-223.

Lilliestam, L. 1996. On Playing by Ear. Popular Music 15 (2): 195-216.

Ley Orgánica. 1990. Ley Orgánica 1/1990, de 3 de octubre. BOE de 4 de octubre, 1990, de Ordenación General del Sistema Educativo. España.

López-Íñiguez, G. y Pozo, J. I. 2014. The Influence of Teachers' Conceptions on Their Students' Learning: Children's Understanding of Sheet Music. British Journal of Educational Psychology 84 (2): 311-28. doi:10.1111/bjep.12026.

Lorenzo Arribas, J. 2011. ¿Dónde están las tocaoras? Las mujeres y la guitarra, una omisión sospechosa en los estudios sobre el Flamenco. TRANS-Revista Transcultural de Música/Transcultural Music Review 15: 1-43.

Marín, C., Pérez Echeverría, M. P., y Hallam, S. 2012. Using the Musical Score to Perform: A Study with Spanish Flute Students. British Journal of Music Education 29 (2): 193-212. doi: 10.1017/S0265051712000046. 
Marín, C., Pérez Echeverría, M. P. y Scheuer, N. 2013. Conceptions of Woodwind Students Regarding the Process of Learning a Piece of Music. Research Papers in Education. Publicación avanzada en Internet. doi: 10.1080/02671522.2013.825310.

Martí, E. y Pozo, J. I. 2000. Más allá de las representaciones mentales: la adquisición de los sistemas externos de representación. Infancia y Aprendizaje 90: 11-30.

McPherson, G. E. 2005. From Child to Musician: Skill Development Turing the Beginning Stages of Learning an Instrument. Psychology of Music 33: 5-35.

Montero, I. y Huertas, J. A. 1999. The Motivational Function of Private Speech in Young Children. Annual Meeting of the AERA, Abril. Montreal, Canadá.

Murphy, D. 1994. Jazz Studies in American Schools and Colleges: a Brief History. Jazz Educators Journal 26: 34-38.

Nielsen, S. G. 1999. Regulation of Learning Strategies During Practice: A Case Study of a Single Church Organ Student Preparing a Particular Work for a Concert Performance. Psychology of Music 27: 218-229.

Ochoa, J. S. 2010. Los discursos de superioridad del jazz frente a otras músicas populares contemporáneas. El Artista 7.

Pêcheux, M. 1978. Hacia el análisis automático del discurso. Madrid: Gredos.

Pedro, J. 2014. Jam Sessions in Madrid's Blues Scene: Musical Experience in Hybrid Performance Models. IASPM@Journal (4) 1: 73-86.

Pelinski, R. 2000. Invitación a la etnomusicología. Quince fragmentos y un tango. Madrid: Akal

Pérez Echeverría, M. P, Postigo, Y. y Marín, C. 2010. Las habilidades gráficas de los estudiantes universitarios: ¿cómo comprenden las gráficas los estudiantes de psicología?. Cultura y Educación 22 (2): 215-229.

Pérez Echeverría, M. P. y Scheuer, N. 2009. External Representations as Learning Tools. En C. Andersen, N. Scheuer, M. P. Pérez Echeverría y E. Teubal Eds. Representational Systems and Practices as Learning Tools. Rotterdam: Sense: 117.

Postigo, Y. y Pozo, J.I. 2004. La representación mental de los mapas geográficos: niveles de procesamiento. Cognitiva, 16: 13-41.

Pozo, J. I. 2008. Aprendices y maestros. La psicología cognitiva del aprendizaje. Madrid: Alianza Editorial.

Pozo, J.I., Scheuer, N., Mateos, M. y Pérez Echeverría, M.P. 2006. Las teorías implícitas sobre el aprendizaje y la enseñanza. En J.I. Pozo et al. Eds. Nuevas formas de pensar la enseñanza y el aprendizaje: Las concepciones de profesores y alumnos. Barcelona: Graó: 95-132.

Real Decreto. 1995. Real Decreto 617/1995, de 21 de abril. BOE de 6 de junio, 1995. Aspectos básicos del currículo del grado superior de las enseñanzas de Música y se regula la prueba de acceso a estos estudios. España.

Sawyer, R. K. Ed. 2006. The Cambridge Handbook of the Learning Sciences. New York: Cambridge University Press.

Schippers, H. 2010. Facing the Music: Shaping Music Education from a Global Perspective. New York: Oxford University Press.

Schön, D. 1987. Educating the Reflective Practicioner. San Francisco, CA: JosseyBass. 
Sloboda, J. A. 2010. Music in Everyday Life: The Role of Emotions. In P. N. Juslin y J. A. Sloboda Eds. Handbook of Music And emotion: Theory, Research, Applications. Oxford: Oxford University Press: 493-514.

Sokol, B. W., Grouzet, M.E. y Ulrich, Müller, U. 2013. Self-Regulation and Autonomy. New York: Cambridge University Press.

Stake, R. 1998. Investigación con estudio de casos. Madrid: Morata. 2006. Multiple Case Study Analysis. New York: The Guildford Press.

Steingress, G. 2004. El flamenco como arte popular y popularizado. En M. López Castro Coord. Introducción al flamenco en el currículum escolar. Sevilla: Universidad Internacional de Andalucía: 181-199.

The Real Book 1978. Jazz Standards. Fifth Edition. Boston.

Torrado J. A. y Pozo J. I. 2008. Metas y estrategias para una práctica constructiva en la enseñanza instrumental. Cultura y Educación 20: 35-48.

Trilla, J. 1997. Relaciones entre la educación formal, la no formal y la informal. En La educación fuera de la escuela. México: Ariel: 187-196.

Trinidad, A., Carrero, V. y Soriano, R. M. 2006. Teoría fundamentada "Grounded Theory". La construcción de la teoría a través del análisis interpretacional. Cuadernos Metodológicos 37. Madrid: Centro de Investigaciones Sociológicas.

Turkenburg, W. 1999. Classical and Jazz Music Compare to World and Pop Music in Music Education. In J.O. Traasdahl Ed. Music Education in a Multicultural Society. Copenhagen: Danish Music Council: 166-173.

Vaughan, V. 2002. Music Analysis in the Practice Room. British Journal of Music Education 19 (3): 255-268. doi: $10.1017 /$ S0265051702000347.

Welch, G., Duffy, C., Potter, J. y Whyton, T. 2006/2008. Investigating Musical Performance (IMP): Comparative Studies in Advanced Musical Learning. Teaching and Learning Research Program. http://www.tlrp.org/proj/Welch.html. Recuperado: 2 Mayo 2014.

Williamon, A., Valentine, E. y Valentine, J. 2002. Shifting the Focus of Attention between Levels of Musical Structure. European Journal of Cognitive Psychology 14: 493-520. 